\title{
Experts vs chercheurs dans la fabrication et la circulation des savoirs et sens sociopolitiques au Sénégal
}

Experts vs Researchers in the Manufacture and Circulation of Knowledge and Socio-political Meaning in Senegal

\section{Boubacar Niane}

\section{(2) OpenEdition Journals}

Édition électronique

URL : http://journals.openedition.org/etudesafricaines/16776

DOI : 10.4000/etudesafricaines. 16776

ISSN : $1777-5353$

Éditeur

Éditions de l'EHESS

Édition imprimée

Date de publication : 8 septembre 2011

Pagination : $549-562$

ISBN : 978-2-7132-2298-6

ISSN : 0008-0055

Référence électronique

Boubacar Niane, "Experts vs chercheurs dans la fabrication et la circulation des savoirs et sens sociopolitiques au Sénégal », Cahiers d'études africaines [En ligne], 202-203 | 2011, mis en ligne le 27 octobre 2013, consulté le 24 juin 2020. URL : http://journals.openedition.org/etudesafricaines/16776 ; DOI : https://doi.org/10.4000/etudesafricaines. 16776

Ce document a été généré automatiquement le 24 juin 2020

(c) Cahiers d'Études africaines 


\title{
Experts vs chercheurs dans la fabrication et la circulation des savoirs et sens sociopolitiques au Sénégal
}

\author{
Experts vs Researchers in the Manufacture and Circulation of Knowledge and \\ Socio-political Meaning in Senegal
}

Boubacar Niane

\section{NOTE DE L'AUTEUR}

Le présent texte s'inscrit dans une recherche en cours et portant sur le degré de prégnance d'une logique de redéfinition de l'État, ou du moins de certaines de ses missions et/ou prérogatives, par contournement, disqualification, décentrement, déconstruction; ceci à partir d'exemples tirés du monde de l'éducation et de la formation. Les experts dont il s'agit dans le texte sont ceux commis pour un laps de temps (mis à disposition), par des organismes internationaux/nationaux (disposant par ailleurs de cadres permanents), pour des études devant aider une prise de décision. Quant aux chercheurs, il s'agit des universitaires. Déjà en 1991, à l'occasion de la cérémonie de présentation des vœux de nouvel An au président de la République, le recteur de l'Université de Dakar déclarait que son institution, en tant que « pôle d'excellence ", avait pour mission de contribuer de manière marquante " à l'élaboration du savoir et à l'épanouissement scientifique et culturel des différentes composantes sociales de la Nation ».

1 Le monde en voie de globalisation peut difficilement se concevoir sans la production et la circulation de savoirs, de sens et de postures fortement homogénéisés, voire standardisés. Faire advenir et maintenir un tel processus, requiert des médiations et des agents pertinents. Et il peut être postulé que des segments, jusque-là opérant dans 
la production des savoirs, devraient être déclassés en conséquence (Bourdieu 1984) et/ ou du moins instrumentalisés en vue de leur utilisation idoine dans le nouvel espace du marché de la connaissance. Mais aussi pour la pérennisation du système qui, comme toute organisation ambitionnant de se perpétuer, se doit de mettre en œuvre une «intégrale des trois $\mathrm{F}$ »: fabriquer (produire) des connaissances, des attitudes et des pratiques; former (adopter un dispositif d'inculcation) des agents aptes à véhiculer lesdites connaissances et pratiques; faciliter (circuler, disséminer) le stock de connaissances constitué (Coblentz 2002).

2 La «dictature " des experts, pour reprendre le mot de Christiane Restier-Melleray (1990), semble avoir fini de s'imposer à l'administration publique de pays en voie de développement, la plupart du temps sous « ajustement structurel ». Par conséquent, une telle situation ne manquerait pas de déteindre, peu ou prou, sur le monde universitaire. Autrement dit, il y aurait un risque, ou déjà une effectivité de la polarisation de l'espace de la recherche universitaire, sa synchronisation avec la nouvelle bulle de l'expertise. Et ceci, au risque de faire perdre au monde universitaire son autonomie essentielle.

3 Certes une telle dynamique n'est pas spécifique à l'Afrique subsaharienne (RestierMelleray 1990 ; George \& Wolf 2002). Mais elle s'y révèle sans doute plus nettement, avec des accentuations dues, dans une large mesure, à la permissivité de l'État et de ses démembrements notamment à partir des années 1980 (Niane 2003), période correspondant à ce que, par euphémisme, des analystes qualifient de « désengagement de l'État ». Sous ce rapport, une constatation majeure peut être faite. L'ambivalence, l'ambiguïté, la variabilité sont présentées (ennoblies) comme les caractéristiques essentielles des «numéros un» de demain du monde de la finance (Goldsmith 1995). Dans l'espace de la recherche, de telles postures sont de plus en plus convoquées pour lire, apprécier les problèmes sociopolitiques notamment des pays africains. Il faut "faire vite ", même au risque de fournir une explication plus ou moins approximative, ou de donner une solution non conforme, inopportune. L'urgence et l'acuité des défis auxquels est confrontée l'Afrique deviennent pour ainsi dire, les référentiels paradigmatiques pour la réflexion et l'action ${ }^{1}$. C'est le règne de l'instantanéité, de l'immédiateté, pour la résolution ( $\mathrm{du}$ moins pour une tentative de recherche) de multiples contraintes auxquelles font face des pays sous-développés et, en particulier leurs populations les plus vulnérables. In fine, toute cette dynamique ne révèle-t-elle pas une tentative de reconfiguration et/ou du moins une redéfinition des principales fonctions de l'État, au Sénégal en particulier, notamment une nouvelle délimitation entre service d'intérêt public et service d'intérêt privé en vue d'un certain rapprochement, voire d'une indistinction entre les deux ?

4 Il est généralement admis que la posture et le temps de la recherche en sciences sociales peuvent difficilement être efficaces sans une certaine distanciation, une déconstruction dans une relative durée. Dans le présent texte, il s'agit de rapprocher ledit temps, du temps de la gestion sociopolitique, socio-économique aux fins d'illustrer un dessaisissement progressif, un processus «d'encadrement» réducteur d'une institution publique comme l'université.

5 La démarche empirique adoptée nous a d'abord amené à inventorier quelques catégories et concepts fondateurs d'une perception et d'une approche pour promouvoir le «développement durable». Ensuite, quelques aspects du processus «d'autodéclassement » des chercheurs universitaires sont analysés, afin de pouvoir esquisser 
les contours d'une valeur, d'une posture suprême que serait l'indistinction pour une réussite de la globalisation.

\section{Promotion de valeurs et démarches « œcuméniques »}

6 Dans le monde en voie de globalisation, il y a nécessité de disposer d'instances de célébration, de légitimation de postures et d'objectifs pour le développement humain ainsi que des segments transnationaux ${ }^{2}$. Lesdits segments dont font partie les " experts ", apparaissent comme des interfaces, des agents devant porter, faire circuler et même imposer lesdits objectifs, leurs rationalités et les implications qui en découlent.

Depuis plusieurs années, une certaine récurrence peut être notée quant à la promotion, pour ne pas dire l'ennoblissement, d'objectifs retenus suite à des « sommets mondiaux sur... » qui tirent leur légitimité entre autres, du nombre de pays participants. Lors de ces grandes rencontres internationales, un certain œcuménisme socio-économique est plus ou moins obtenu. Subséquemment, les États et les organisations multilatérales pertinentes sont mis " en ordre de bataille " pour vulgariser et atteindre les objectifs dessinés. C'est ainsi par exemple qu'il y a une évolution bien nette dans les conceptions d'intervention attendues (fortement suggérées) des acteurs non étatiques. De la posture «ignorer l'État», l'on justifiera ensuite la recommandation «travailler avec l'État", avant de demander de "travailler avec les acteurs décentralisés». À la conférence de Jomtien (1990), on célèbre le partenariat : l'État doit ouvrir des espaces de concertation avec des segments, notamment les collectivités locales, le secteur privé. À Dakar en 2000, la conférence sur l'« Éducation pour tous " recommande fortement l'équité, la facilitation de l'accès à l'école pour tous les enfants. Quant au sommet sur les Objectifs du millénaire pour le développement (OMD) de 2000, la réduction de la pauvreté (sous tous ses aspects, en particulier en matière de santé, d'éducation, de protection de l'environnement) devient la priorité pour 2015. À Genève en 2001, l'éducation est encore ciblée en préconisant, entre autres, une dynamique partenariale impliquant la société civile (Petit \& Comhaire 2010). D'autres thèmes «fédérateurs» illustrent bien ce processus ou tentative d'homogénéisation des politiques socio-économiques. C'est le cas de la «bonne gouvernance», ou de «l'État régulateur » avec les DSRP (Document de stratégie de réduction de la pauvreté) initiés par la quasi-totalité des pays africains subsahariens.

8 Entre autres rationalités mises en exergue pour obtenir l'adhésion du plus grand nombre, l'on peut noter l'urgence et la nécessité d'une «implication solidaire ». C'est au nom de cette solidarité que l'on se mobilise en faveur des populations les plus affectées. Une autre rationalité (quasiment jamais évoquée explicitement) demeure la capture de financements. En effet, ces campagnes mondiales de solidarité permettent de mobiliser d'énormes moyens financiers. Dès lors, un marché de l'utilisation desdits moyens s'organise, avec des enjeux, des acteurs développant des stratégies efficaces en vue d'en profiter au maximum.

9 Comme un corollaire de cette recherche de profits symboliques et/ou financiers, il se développe des sortes de recherches appropriées. Parmi celles-ci, la « recherche-action » reste la plus valorisée, avec une philosophie, des principes, un champ d'application. Elle émerge dans des contextes de tension, de changement. Face à des urgences, elle doit trouver des réponses «en temps réel» en vue de modifier une situation initiale de 
« crise ». En cela, elle peut être considérée comme une aide à la prise de décision. Dans la recherche-action, on privilégie l'interaction entre chercheurs professionnels et acteurs de la société ciblée en vue d'une construction commune et partagée des connaissances grâce notamment à une immersion peu longue (démarche ethnographique). Ce sont des "recherches appliquées qui utilisent des savoirs constitués préalablement à la rencontre des acteurs de terrain» (Hugon \& Pastiaux 1987). Au terme d'une étude consacrée à la recherche-action, Gabriel Goyette et Michelle Lessard-Hébert (1987) notent que les différents types y afférents se réfèrent, s'appuient sur « cinq langages épistémologiques : le langage cartésien ou positiviste, la praxéologie, l'approche systémique, les approches compréhensives, la dialectique ». Ce qui ne jure pas avec la théorie de la pédagogie progressiste, de la conscientisation et de la libération développée par Paulo Freire (1974), quand bien même ce dernier convoque d'autres référentiels philosophico-politiques et s'inscrit dans la durée pour obtenir une émancipation des populations ciblées par l'action et/ou la formation.

10 Le troisième élément du dispositif de promotion-imposition de valeurs et postures fortement homogénéisées est représenté par les experts. Si ces derniers, comme les chercheurs, disposent d'une somme de connaissances spécifiques avérées, leurs statuts, fonctions, postures et images peuvent suffisamment différer.

11 Le chercheur est autorisé à explorer le « réel » afin d'y découvrir éventuellement des régularités, des systèmes de relations, etc. (Bourdieu 1967). Ses «vérités» et conclusions relèveraient plutôt du relatif, du provisoire, de la discussion entre membres d'une communauté de scientifiques partageant des normes et autres procédures de travail, d'investigation.

12 Quant à l'expert, il est expressément commis à "certifier»: autorité de la chose expertisée (autorité de la chose jugée). Ses verdicts sont à appliquer. C'est comme le règne de l'absolu d'une parole autorisée. L'expert apparaît dès lors comme un néodémiurge, suite à une sorte de transmutation des «experts de l'État-national », de la bureaucratie étatique (Dreyfus 1976) en des experts méta-étatiques. Mais pour l'essentiel, les deux figures d'agents (experts) tirent leur légitimité du même principe fondateur; seuls changent le commanditaire, le lieu d'exercice et la temporalité contractuelle : «contrat à durée indéterminée » (CDI) pour les premiers, "contrat de louage de services » éphémère, épisodique, fugace pour les seconds.

13 À l'expert, il est dévolu un pouvoir dont le fondement est son «caractère rationnel, reposant sur la croyance en la légalité des règlements arrêtés et du droit de donner des directives qu'ont ceux qui sont appelés à exercer la domination par ces moyens (domination légale)» (Weber 1971: 222). C'est parce que le savoir du néo-expert apparaît comme un "savoir spécialisé ", reconnu et recherché par les organisations transnationales, qui n'aurait comme déterminant et ambition que la seule science/ technique, la production de biens de la connaissance, pour le « salut » (rédemption) des populations, objets et bénéficiaires du «logos» dudit expert. Pour paraphraser Marc Poncelet (2010), l'expert préconise, propose des «IAE ( (inventions assistées par expert) à l'instar de la PAO (publication assistée par ordinateur). Il reste un émetteur, un producteur de sens ; il est un cadre entendu comme relais, connecteur devant pouvoir se mouvoir bien facilement dans la "Cité des projets " en multipliant notamment ses "connexions» (large réseau), en ayant une "bonne réputation», en étant un «donneur de souffle» afin de toujours satisfaire ses «clients» (Boltanski \& Chiapello 1999). En somme, l'expert doit toujours préserver et conforter son employabilité3. 


\section{d'homogénéisation :}

- expert de légitimation/expert d'imposition ;

- expert universitaire (chercheur-expert à la demande)/expert freelance ;

- expert national/expert international, etc.

En outre, le monde de l'expertise demeure un milieu relativement stressant. Il s'y développe une très forte concurrence, sans compter l'incertitude quant à l'obtention des commandes et des contrats. Aussi, des ruses et des astuces y sont nécessaires afin d'apparaître scientifique tout en cherchant à faire advenir les "présomptions", les attentes du commanditaire. C'est ainsi par exemple que pour faire dire aux statistiques ce dont les instances dominantes (au niveau national) et méta-étatique veulent disposer pour fonder leurs "décisions" ou accepter certaines «suggestions", les experts s'adonnent souvent à un jeu très « lucratif » pour eux : manipuler les données en vue de présenter des statistiques qui, quand bien même elles seraient erronées, n'en demeurent pas moins " pertinentes » à souhait. Ainsi, ils peuvent espérer disposer d'un capital (spécifique) de suggestion, voire de prescription d'une part, multiplier les «missions » avec tous les avantages qui y sont liés d'autre part. Parce qu'en tant qu'agents de l'espace transnational, lesdits experts ont intérêt à conserver leur « pot de miel » (positions-avantages-privilèges). Entre autres exemples de discours (des agents des institutions transnationales) modulé selon le destinataire, Davison Budhoo, ancien expert du Fonds monétaire international (FMI), cite le cas d'un pays sous « ajustement » où, grâce à des glissements " conscients ", une vérité est transfigurée et servie selon les spécificités et les instances de décision et/ou d'exécution concernées. À ce propos, il confesse «[...] comme le pays continue à résister à notre médication toujours plus mortelle qui le met en état de se tenir prêt à un arrangement avec nous, nous continuons à recourir aux méfaits et à la fausse information, nullement intimidés, afin de le mettre à genoux. Parmi plusieurs méfaits, nous avons influencé la Banque mondiale, apparemment en dépit de la juste appréciation de son propre personnel de mission, afin de positionner nos propositions de politiques pour le pays " (Budhoo $1990: 4)$.

16 Avec de tels aveux ou dénonciations, la carrière du pourfendeur peut être brisée, de même que sa conscience (ibid.) ${ }^{4}$. Par conséquent, les experts peuvent, comme dans la situation des journalistes analysée par Pierre Bourdieu (1996), devenir "féroces", condescendants, pour ne pas dire méprisants, par rapport à ceux-là qu'ils sont chargés d'étudier, de rapporter.

17 De nos jours et pour ce qui concerne le Sénégal notamment, parmi les domaines d'investigation pour lesquels sont le plus sollicités les experts, il y a le management des institutions et organisations, les nouvelles techniques administratives, la macroéconomie, le développement local, l'économie de l'éducation et de la santé. En d'autres termes, ce sont là des thématiques en dehors desquelles les possibilités de contrats demeurent assez limitées. Ce qui peut surdéterminer les orientations et les programmes de recherche d'institutions publiques qui sont souvent relativement démunies en termes de moyens matériels et financiers. 


\section{Universitaires sous influence}

dévoiler un processus de mise au pas, voire de déclassement des chercheurs par rapport aux experts: dessaisissement, en particulier, des spécialistes en sciences sociales ou plutôt leur relégation au rang d'agents d'amplification, de vulgarisation et/ ou de célébration (sanctuarisation) de nouveaux savoirs et acteurs. Autrement dit, les objets d'étude et d'analyse des universitaires leur seraient peu ou prou imposés par des institutions transnationales, par le biais de segments transnationaux que seraient les experts, certaines organisations (dont les $\mathrm{ONG}$ ), mais toujours grâce à des procédures et moyens relativement complexes et indirects.

19

Si cela advient, c'est parce que, dans une large mesure, la recherche universitaire au Sénégal présente une faiblesse structurelle certaine. Ce handicap faciliterait non seulement une synchronisation, mais aussi une relative dépendance (vassalisation) ou tout au moins une propension de la part des universitaires à "re-travailler» des thèmes et modalités de recherches pré-positionnés, déjà ennoblis, sanctifiés par des segments transnationaux (Niane 2000).

La productivité scientifique des enseignants et chercheurs de l'UCAD (Université Cheikh Anta Diop) reste relativement faible. En 2006, les 1110 enseignants/chercheurs de l' UCAD étaient ainsi répartis : professeurs et directeurs de recherche, 162 ; maittres de conférences et chargés de recherches, 173; maîtres d'enseignement et maîtres assistants, 416; assistants et assistants de recherche, 280; chefs de travaux et professeurs certifiés, 79. Sur une période de trois ans (2001-2004), les publications de ce personnel s'élèvent à 2967 articles et ouvrages ${ }^{5}: 1558$ en sciences médicales ; 766 en sciences biologiques, agronomiques et vétérinaires; 246 en lettres et sciences humaines ; 97 en sciences juridiques et politiques ; 57 en sciences économiques ; 108 en sciences de la terre, des milieux aquatiques, des climats; 83 en sciences ingénierie physique et chimie ; 52 en mathématiques (UCAD 2006). En moyenne, cela représente 989 publications par an, soit environ 0,9 publication par an et par chercheur ${ }^{6}$.

21 Plusieurs facteurs, d'ordres matériel, infrastructurel et organisationnel, seraient au principe d'une telle situation. Les rémunérations sont jugées insuffisantes, notamment par les jeunes enseignants. Malgré la mise en place d'une nouvelle bibliothèque centrale bénéficiant des TIC (entre autres, une documentation en ligne), l'accès aux récentes publications reste assez difficile. Les bibliothèques de département sont relativement démunies quand elles existent. Il s'y ajoute une faible visibilité des universitaires dans l'édition. Tandis qu'en Europe par exemple, maints universitaires sont directeurs de collections, les universitaires sénégalais ne le sont quasiment pas, du fait même de la faiblesse de l'édition. La modicité des crédits internes de recherche apparaît comme un autre facteur négatif. Il est aussi assez rare de trouver des programmes généraux de recherches de départements. Les recherches plus individuelles que celles des groupes, sont réalisées en fonction des réseaux de relations personnelles de l'universitaire (Niane 2009).

22 C'est certainement pourquoi Jean Copans (2010:5) ne doute pas «qu'en un peu plus d'un demi-siècle les sciences sociales africaines n'ont pas encore réussi à atteindre le seuil critique d'une autonomie intellectuelle pleine et entière ». Pour lui, ce qui affaiblit les sciences, pas seulement en Afrique, reste «le poids de plus en plus pesant des recherches de consultance et d'application immédiate. En Afrique noire, la soi-disant 
recherche sur le développement mobilise toutes les énergies et produit des clivages insurmontables, de moins en moins remis en cause, entre une dynamique qui serait le contrôle direct des chercheurs ou de leurs institutions nationales et une "marée" d'appels d'offres à court terme aux seules finalités administratives ou alimentaires " (ibid. : 10). Cette " asphyxie ${ }^{7}$, selon l'auteur, accélérerait largement le phénomène de " fuite de cerveaux » observé notamment chez les universitaires sénégalais (Niane et al. 2003). Les médiations usitées ou facilitant l'avènement de tous ces effets observés sont capables d'influencer très fortement les universitaires. Elles sont multiples et s'inscrivent dans des systèmes dont l'efficacité est largement fonction de leur capacité d'influence par touches homéopathiques.

De nombreux « forums de discussions " sont mis en ligne sur l'Internet par des ONG et autres organisations similaires afin d'y débattre entre autres, des approches, des thèmes et zones d'intervention prioritaire, etc. Ici, l'instantanéité et le nombre de participants seraient comme la valeur et la vérité de référence. Plus le nombre de personnes échangeant presque simultanément (en «temps réel ») sur un thème (peu importent le statut et les desseins de l'initiateur) est élevé, plus le sujet est digne d'intérêt, avec de nouveaux enjeux et des agents prêts à s'investir, pour apporter leur "contribution", quand en réalité il participe à la consolidation d'un espace de jeu social (Bourdieu 1984). Et la superposition (sédimentation) des instantanéités finit par avoir un effet structurant auquel les chercheurs peuvent difficilement échapper. Par exemple, l'économie de l'éducation est en voie d'occuper une position centrale dans le domaine de l'expertise pour des pays comme le Sénégal. Les universitaires sont bien sollicités, encouragés à s'investir dans des recherches. Ce qui est une illustration d'une perte relative d'autonomie de pensée et de choix de thèmes de réflexion; pas au sens d'enfermement du chercheur, plutôt de perte d'identité, de non prise de responsabilité suffisante (Bourdieu 1975).

En outre, la plupart des ong intervenant au Sénégal font partie de "Tables de concertation $»^{8}$. Ces structures permettent, entre autres, à leurs membres de contribuer à la définition, à la mise en œuvre et à l'évaluation de politiques de développement socio-économique. Elles sont des sortes de contre-pouvoir par rapport à l'omnipotence de l'État-central. Sous ce rapport, elles ne manquent pas de mener des campagnes de plaidoyer et de lobbying auprès d'acteurs pertinents (Niane 2003). Et de plus en plus d'universitaires sont animateurs d'oNG et/ou sont sollicités par ces dernières en vue de valoriser un tant soit peu leur image scientifique (ibid. 2000).

Les réunions des groupes de travail entre représentants de l'administration publique (dont l'université) et experts sont d'autres moments pour une imposition douce de catégories et de concepts divers. L'observation desdites rencontres permet de noter des argumentaires très efficaces, des techniques de persuasion avec divers référentiels pour convaincres.

26 La domination des universitaires par «l'urgence» se manifeste également par une acceptation tacite d'une « recherche instantanée ». Cela peut advenir, d'une part, en comprimant le temps nécessaire de la recherche afin de le faire coïncider avec les exigences des délais relativement courts du commanditaire et, d'autre part, en acceptant de se soumettre au temps des médias. Ces derniers, en sollicitant les chercheurs, leur demande assez souvent, non seulement d'être des "prophètes du social » en expliquant ou en prédisant sans recul ni distanciation, mais aussi et surtout en opérant des « dédoublements à la carte ». Le même chercheur ("pluri-spécialiste ») 
attitré $^{10}$ par une chaîne de radio ou de télévision intervient soit comme sociologue ou politologue, soit comme psychosociologue ou économiste. Des exemples notoires ont ainsi suscité des réactions relativement indignées de chercheurs. Sous ce rapport, le journaliste sociologue Pathé Mbodje (2009), observe « la dictature de la dépêche pour ceux appelés à commenter des faits de société qu'ils ne prennent pas le temps de conquérir, de construire et de constater, faute de recul scientifique nécessaire » et se méfie donc des "affirmations à l'emporte-pièce des acteurs des plateaux de télévision ". Quant à Aboubacar Abdoulaye Barro (2009), après avoir constaté que «depuis quelque temps, les plateaux de télévision et les radios sont pris d'assauts par de drôles de spécialistes : dans la plupart des cas ce sont des sociologues ", il admet qu'il serait «moins choqué si les invités dans ce type de débat parlaient simplement en tant que citoyens; mais il est intellectuellement douteux de profiter d'un titre académique acquis à travers une thèse de doctorat pour s'inviter dans tous les plateaux de télévision et professer des banalités ».

Pour tout dire, le «chercheur en temps réel» devient de facto un expert-consultant dont le "discours autorisé » par les médias est en même temps légitimé par ces derniers qui le portent et l'amplifient à souhait. L'on peut valablement postuler une homologie, ou en tout cas une forte ressemblance, entre les effets structuraux notés par Pierre Bourdieu (1975) en ce qui concerne la télévision et le champ intellectuel français et ceux que l'on observe entre l'expertise et le champ universitaire dans des pays comme le Sénégal. Et l'on assiste donc à des effets d'annonce de la certification (ou de l'invalidation) de mesures et/ou de comportements, par de doctes voix. Or, si l'on en croit Christiane Restier-Melleray (1990), «l'expertise telle qu'elle est pratiquée aux États-Unis [très souvent cités en référence] constitue le mode de régulation d'un modèle institutionnel spécifique fondé sur le pluralisme des centres de décision et la séparation des pouvoirs. C'est une procédure inscrite dans un système reposant sur le principe de mises en œuvre de négociations antérieures à la prise de décision, dans lequel les choix politiques doivent être légitimés au coup par coup. Le rôle de l'opinion publique y est donc déterminant et la transparence des décisions une obligation théorique ». L'expertise d'aujourd'hui, en particulier celle servie aux pays en voie de développement, serait comme un « avatar volontariste du saint-simonisme » (ibid.). Car on assiste plutôt à un processus tronqué. Un mouvement de translation théorique et pratique (de la procédure exigeant des négociations) est opéré par l'imposition d'une seule voix, d'une seule partie. Ce qui ne manque pas d'influencer la communauté des chercheurs qui, par effet de contagion, se trouve peu à peu ébranlée dans ses postures et croyances (doxa et fides). Elle peut, dès lors, se retrouver, peu ou prou, arrimée à un mouvement de balancier, tiraillée entre respect des normes scientifiques et règne du scoop médiatique.

Enfin, l'endossement par l'université de programmes de formation élaborés par des institutions d'organismes transnationaux est une illustration presque parfaite d'un relatif «embrigadement». C'est le cas, par exemple, de la "formation en politiques sectorielles et gestion des systèmes éducatifs (PSGSE) $»^{11}$. Pour l'essentiel, les modules ont été conçus par les experts de l'Institut de formation de la Banque Mondiale et validés suite à des rencontres et séminaires avec les universitaires en particulier. Insérée dans le système LMD (licence, master, doctorat) ${ }^{12}$, cette formation mise en œuvre par le Pôle d'analyse sectorielle en éducation de Dakar, est institutionnellement rattachée à la Faculté des sciences et technologies de l'éducation et de la formation 
(FASTEF) de l'Université de Dakar ${ }^{13}$. Ce sont peut-être les prémisses d'un processus d'intervention directe des organisations transnationales dans la formation actuellement dévolue aux universités, ou tout au moins une co-action plus subie que contrôlée par le monde universitaire, et dont l'un des fondements serait la fin de la délimitation, ou du moins l'estompement progressif des frontières entre les statuts, les domaines de compétences d'institutions, d'agents jusque-là bien spécifiés.

$\mathrm{Au}$ demeurant, l'indistinction entre expert et chercheur n'est pas un exemple unique dans l'espace de l'enseignement supérieur au Sénégal. Elle semble devenir comme une valeur suprême, un référentiel paradigmatique. C'est ainsi qu'elle s'applique de plus en plus entre les universités, les grandes écoles, les instituts universitaires et les instituts supérieurs non universitaires; mais aussi entre les services d'intérêt public et ceux d'intérêt privé (Niane 2011).

31 Contrairement à la Conférence de Berlin (1881), le processus de globalisation en cours semble s'orienter vers une nouvelle redistribution de "colonies» d'une nécessaire virtualité mais qui n'en demeurent pas moins «fonctionnelles». Pour paraphraser L. Althusser (1975), l'on pourrait parler de l'ouverture d'un «nouveau continent » de savoirs et de sens devant fonctionner et produire les effets attendus. Autrement dit, avec ce changement paradigmatique, l'on peut mesurer la durabilité, l'efficacité des impositions cognitives, catégorielles d'une domination plus symbolique que physique, directe comme dans le système politique et administratif de la période coloniale. Dans cette configuration homogénéisante, les experts apparaîtraient comme des néoexplorateurs, des néo-évangélisateurs. Par leur intercession, des savoirs et des sens ainsi que des approches sur le développement socio-économique et politique sont proposés pour lire la situation de pays comme le Sénégal. Et ceci grâce notamment à la promotion de la logique de l'urgence avec, entre autres conséquences, des chercheurs peu ou prou confinés à des positions de faire-valoir, de cautionnement de procédures, de postures privilégiées. À cela s'ajoutent la porosité entre les espaces ainsi qu'une forte mobilité, pour ne pas dire ambivalence, des agents mobilisés ou mobilisables dans ce qui pourrait être un processus de reconfiguration de l'État (et de ses agents) ou en tout cas, d'une redéfinition de leurs principales missions et fonctions.

\section{BIBLIOGRAPHIE}

ALthUSSER, L. 1975 Pour Marx, Paris, Maspero.

BARRO, A. A. 2009 « Qui sont ces nouveaux sociologues? », Sud Quotidien, Dakar, 3 déc.

BOLTANSKI, L. \& CHIAPELLO, E. 1999 Le nouvel esprit du capitalisme, Paris, Gallimard.

BOURDIEU, P. 1967 Métier de sociologue. Préalables épistémologiques, Paris, Éditions de l'EHESS.

- 1975 « Les intellectuels dans le champ de la lutte des classes », La nouvelle critique, 10 (87) : 66-69.

- 1984 Questions de sociologie, Paris, Éditions de Minuit.

- 1996 Sur la télévision, suivi de L'emprise du journalisme, Paris, Éditions Liber. 
BUDHOO, D. L. 1990 Enough is Enough... Dear Mr Camdessus... Open Letter of Resignation to the Managing Director of the International Monetary Fund, New York, New Horizons Press.

COBLENTZ, J. B. 2002 « Organizational Sustainability : The Three Aspects that Matter », Exposé pour la $1^{\text {re }}$ session d'orientation stratégique du Réseau ouest-africain de recherches en Éducation (ROCARE) à Dakar, Washington D.C., Academy for Educational Development.

COPANS, J. 2010 Un demi-siècle d'africanisme africain. Terrains, acteurs et enjeux des sciences sociales en Afrique indépendante, Paris, Karthala.

DREYFUS, F. 1976 « La professionnalisation dans l'administration : recherches sur la fonction idéologique de l'expertise ", Paris, Annuaire international de la fonction publique 1975-76 : 295-309.

FREIRE, P. 1974 Pédagogie des opprimés, suivi de Conscientisation et révolution, Paris, Maspero.

GEORGE, S. \& WOLF, M. 2002 La mondialisation libérale, Paris, Grasset.

GOLDSMITH, J. 1995 « Éloge du prédateur », Monde diplomatique, mai : 20.

GOYETTE, G. \& LESSARD-HÉBERT, M. 1987 La recherche-action : ses fonctions, ses fondements et son instrumentation, Montréal, Presses de l'Université du Québec.

HUGON, M.-A. \& PASTIAUX, G. 1987 « Recherches impliquées, recherches-actions : Le cas de l'éducation (Compte rendu de colloque) », Revue française de pédagogie, 79 : 113-116, <http://www. persee.fr/web/revues/home/prescript/article/rfp_05567807_1987_num_79_1_2425>.

MBODJE, P. 2009 « De la sociologie et des (nouveaux ?) sociologues au Sénégal. La crise de l'écrit ne doit pas signifier la crise de la sociologie », Kotch, Dakar, 51, 15 déc. ; 52, 16 déc.

NIANE, B. 2000 « Permissivité d'un espace de formation : le cas de l'Université de Dakar », in Y. LEBEAU \& M. OGUNSANYA (eds.), The Dilemma of Post-Colonial Universities, Ibadan, IFRA/ABB : 301-315. - 2003 « Du gouvernement des ONG au Sénégal ? », in Y. LEBEAU, B. NIANE, A. PIRIOU \& M. DE SAINT MARTIN (dir.), État et acteurs émergents en Afrique, Paris, Karthala : 87-108.

- 2009 « Entre public et privé : de quelques mutations dans l'espace de l'enseignement supérieur au Sénégal »,Écritures plurielles, Revue semestrielle d'études universitaires, Dakar, FASTEF-UCAD : 145-161.

- 2011 « Les EPES au Sénégal, entre mission de service public et entreprenariat éducatif », in M. LECLERC-OLIVIER, G. SCARFÒ GHELLAB \& A.-C. WAGNER (dir.), Les mondes universitaires face aux logiques du marché : circulation des savoirs et pratiques des acteurs, Paris, Karthala.

NIANE, B., NDIAYE, F. \& SOUGOU, O. 2003 Universités et transformations sociales : le cas de l'Université Cheikh Anta Diop de Dakar et de l'Université Gaston Berger de Saint-Louis, Londres, Centre for Higher Education Research and Information, Open University.

PETIT, P. \& comhaire, G. 2010 « Les associations de la société civile et le partenariat éducatif », in P. PETIT (dir.), Société civile et éducation. Le partenariat à l'épreuve du terrain, Louvaint-La-Neuve, Academia-Bruylant : 15-47.

PONCELET, M. 2010 « Vers un deuxième âge de la nébuleuse des Organisations de la Société civile au Sud », in P. PETIT (dir.), op. cit. : 215-223.

RESTIER-MELLERAY, C. 1990 « Experts et expertise scientifique. Le cas de la France », Revue française de science politique, $4: 546-585$.

UNIVERSITÉ CHEIKH ANTA DIOP (UCAD) 2006 « Interface », Journal bimensuel de l'UCAD, 3, Dakar, sept.oct.

WEBER, M. 1971 Économie et société, Paris, Plon. 


\section{NOTES}

1. Par exemple les orientations sociopolitiques suggérées à des pays sous ajustement structurel par la Banque Mondiale et relatives à la non-rentabilité économique des secteurs de l'éducation et de la santé. Quelques années plus tard, l'institution a été obligée de revenir sur une telle position.

2. Nous désignons comme "segments transnationaux» des agents (organismes, individus, groupes d'individus, etc.) qui, par-delà les frontières nationales, partagent les mêmes valeurs, priorités, goûts, normes, etc. Ils peuvent donc jouer un rôle d'interface pour la transmission et l'imposition de postures et rationalités homogénéisées.

3. « [...] capacité dont les personnes doivent être dotées pour que l'on fasse appel à elles sur des projets [...]. Celle-ci est le capital personnel que chacun doit gérer et qui est constitué de la somme de ses compétences mobilisables " (BOLTANSKI \& CHIAPELLO 1999: 144).

4. Ce qui est arrivé à Davison Budhoo, dont l'ouvrage n'a pu être édité et diffusé que grâce notamment à l'engagement d'une coopérative d'organisation syndicale anglaise. L'auteur révèle aussi d'autres pressions qu'il aurait subies, sans compter la quasi impossibilité à trouver un nouvel emploi.

5. Les statistiques du rectorat ne font pas la distinction entre articles et ouvrages. Cependant, il nous semble que les premiers sont très nettement plus nombreux. Il serait aussi intéressant de connaître la répartition de la production scientifique selon les grades. L'on peut valablement présumer que le ratio nombre publications/ enseignant-chercheur est plus important parmi les candidats aux listes d'aptitude du CAMES (Conseil africain et malgache de l'enseignement supérieur). Autrement dit, les universitaires publieraient davantage pour les besoins de leur promotion personnelle.

6. À notre connaissance, il n'existe pas de statistiques officielles sur le nombre de rapports de consultance élaborés par des universitaires sénégalais. Il serait intéressant de pouvoir le comparer au nombre de publications scientifiques. En tout état de cause, bien des indices laissent penser que ce nombre de rapports de consultance par des universitaires croît assez rapidement depuis quelques années.

7. À ce propos, les stratégies possibles (avec des risques certains) de jeunes chercheurs sélectionnés par le Programme de petites subventions initié par le ROCARE (Réseau ouest et centre africain de recherche en éducation) avec l'appui financier du CRDI (Centre de recherches pour le développement international), de l'UEMOA (Union économique et monétaire de l'Ouest-africain), des Pays-Bas. Entre autres conditions, pour bénéficier d'une subvention de 3 millions de Francs CFA, les équipes de chercheurs doivent se conformer au thème général de recherche "imposé » pour chaque cycle de programme, <www.rocare.org $>$.

8. Voir par exemple la "Plateforme des acteurs non étatiques» au Sénégal, $<$ www.plateforme-ane.sn>.

9. Nous menons actuellement des observations et entretiens sur ces procédures.

10. Après avoir adoubé le sociologue $M$. Ndiaye, les médias ont actuellement jeté leur dévolu sur D. Diakhaté, K. Niang et A. K. Diao.

11. Le programme est soutenu par plusieurs partenaires: Agence universitaire de la Francophonie, Institut de formation de la Banque Mondiale, Secrétariat de l'initiative 
Fast Track de la Banque Mondiale, Conférence de ministres de l'Éducation (CONFEMEN/ PASEC), Institut international de planification de l'Éducation, Institut statistique de l'Unesco, Organisation internationale de la Francophonie, <www.poledakar.org>.

12. Voir le protocole d'accord entre l'UCAD et le Bureau régional de l'Unesco à Dakar.

13. <www.fad-fastef.org>.

\section{RÉSUMÉS}

\section{Résumé}

Par l'intercession de segments transnationaux, des savoirs, des sens ainsi que des approches sur le développement socio-économique et politique sont proposés pour comprendre la situation du Sénégal. De même, de nouveaux acteurs, en particulier les experts et/ou la société civile, sont présentés comme les plus aptes à contribuer à l'émergence d'une démocratie citoyenne, d'un développement socio-économique durable.

Cette contribution tente de décrypter les divers processus et médiations par lesquels advient cette promotion/émergence qui semble illustrer un processus de dessaisissement des spécialistes des sciences sociales, ou plutôt leur relégation au rang d'agents d'amplification, de vulgarisation et/ou de célébration (sanctuarisation) de nouveaux savoirs et acteurs. Autrement dit, les objets d'étude et d'analyse des chercheurs, en sciences sociales notamment, leur seraient peu ou prou imposés par des médiations et médiateurs «trans-nationalisés ", homogénéisés. Et, par-delà cet «encadrement», ne se dessine-t-il pas, in fine, un processus de redéfinition de l'État, en particulier de la notion de service public?

Abstract

Through the intercession of transnational segments, knowledge, meaning, approaches on socioeconomic and policy development are proposed to understand the situation of Senegal. Likewise, new players, especially the experts and/or civil society, are presented as the most likely to contribute to the emergence of democratic citizenship, of sustainable socio-economic development.

This paper attempts to analyze the various processes and mediations by which this emergence seems to illustrate a process of disenfranchisement of social scientists or if anything, their downgrading to the roles of dissemination and canonization officers of new knowledge and new players. Does that new construct, more or less imposed by "trans-nationalized", homogenized mediations and mediators, not foreshadow a process of redefinition of the State, particularly the notion of public service?

\section{INDEX}

Mots-clés : Sénégal, chercheurs, État, experts, indistinction, production, savoirs, transnationalisation

Keywords : Senegal, Researchers, State, Experts, Indistinctness, Production, Knowledge, Transnationalization 
AUTEUR

BOUBACAR NIANE

Faculté des sciences et technologies de l'éducation et de la formation, Université Cheikh Anta Diop, Dakar, Sénégal. 